\title{
Reduction of monocyte chemoattractant protein 1 and macrophage migration inhibitory factor by a polyphenol-rich extract in subjects with clustered cardiometabolic risk factors
}

\author{
Lysette N. Broekhuizen ${ }^{1}$, Diederik F. van Wijk ${ }^{1}$, Hans Vink ${ }^{2}$, A. Stalmach ${ }^{3}$, A. Crozier ${ }^{3}$, B. A. Hutten ${ }^{1}$, \\ John J. P. Kastelein ${ }^{1}$, Paul G. Hugenholtz ${ }^{4}$, Wolfgang Koenig ${ }^{5}$ and Erik S. G. Stroes ${ }^{1 *}$ \\ ${ }^{1}$ Department of Vascular Medicine, Academic Medical Center, 1105 AZ Amsterdam, The Netherlands \\ ${ }^{2}$ Department of Physiology, University of Maastricht, Maastricht, The Netherlands \\ ${ }^{3}$ Centre for Population and Health Sciences, University of Glasgow, Glasgow, UK \\ ${ }^{4}$ Department of Cardiology, Erasmus University, Rotterdam, The Netherlands \\ ${ }^{5}$ Department of Internal Medicine - Cardiology, University of Ulm Medical Center, Ulm, Germany
}

(Received 5 July 2010 - Revised 28 March 2011 - Accepted 30 March 2011 - First published online 28 June 2011)

\section{Abstract}

Inflammation is a hallmark of the metabolic syndrome, which also contributes to a pro-atherogenic state. NF-кB activation, a critical step in regulating inflammatory reactions, can be inhibited by polyphenol (PF) extracts, at least in vitro. In the present study, we set out to study whether a PF-rich extract could attenuate the chronic inflammatory state and/or an acute immune response in vivo in subjects with clustered metabolic risk factors. A commercially available, PF-rich extract (500 mg daily) or placebo was administered for 4 weeks to thirty-four subjects with two or more metabolic risk factors using a randomised, double-blind, cross-over design. During the final study visit, an acute inflammatory challenge (lipopolysaccharide (LPS) $1 \mathrm{ng} / \mathrm{kg}$ body weight) was administered to a random subgroup of subjects (PF-rich extract $(n 12)$ and placebo $(n 12)$ ). The PF-rich extract modestly reduced the inflammatory chemokines monocyte chemoattractant protein 1 (MCP-1) and macrophage migration inhibitory factor (MIF) (MCP-1 - 6.5\% (PF, median 116 (interquartile range 97-136) pg/ml $v$. placebo, median 124 (interquartile range 105-153) pg/ml; $P<0 \cdot 05$ ); MIF - 10.8\% (PF, median 2512 (interquartile range 1898-3972) pg/ml $v$. placebo, median 2814.5 (interquartile range $2296-3852) \mathrm{pg} / \mathrm{ml} ; P<0.05$ ); however, other measured markers of inflammation and cardiometabolic disease, such as C-reactive protein, IL-6, HDL-cholesterol, adiponectin and oxidised LDL, remained unaffected. Following the LPS challenge, we found a statistically significant $48 \%$ reduction of MCP-1 production in the PF-rich extract group ( $n$ 12) $v$. placebo $(n 12)$ over $6 \mathrm{~h}$ (PF 766 (SD 155) $v$. placebo 1466 (SD 989) ng/ml; $P<0 \cdot 05$, area under the curve). In conclusion, short-term oral administration of the PF-rich extract caused a modest anti-inflammatory effect in subjects with clustered metabolic risk factors. Further dose-ranging studies are needed to evaluate whether and to what extent PF-rich extracts can be used to reduce the pro-inflammatory state in subjects with metabolic diseases at increased cardiovascular risk.

Key words: Flow-mediated dilation: Polyphenols: Inflammation: Cardiometabolic risk factors: Monocyte chemoattractant protein 1

Inflammation plays a critical role during all stages of the atherogenic process, ranging from the initiation of endothelial dysfunction to the onset of a cardiovascular event ${ }^{(1)}$. In agreement with this, elevated levels of pro-inflammatory chemokines and cytokines have been associated with increased cardiovascular risk $^{(2-5)}$. Novel interventions that attenuate inflammation are being evaluated as an add-on strategy in patients at increased cardiovascular risk ${ }^{(6)}$. The metabolic syndrome, a major risk factor for CVD, is characterised by a chronic low-grade inflammatory state ${ }^{(7-9)}$. The latter has been attributed to inappropriate adipocyte enlargement accompanied by increased stress in the endoplasmatic reticulum of the adipocytes, leading to activation of NF- $\mathrm{B}$ and subsequent inflammatory activation as attested by an increased production of IL- 6 , TNF- $\alpha$, macrophage migration inhibitory factor (MIF) and monocyte chemoattractant protein 1 (MCP-1) ${ }^{(10,11)}$. These pro-inflammatory cytokines modulate the paracrine function of the adipocytes, further contributing to systemic inflammation and insulin resistance in patients with obesity ${ }^{(10,12)}$.

Polyphenols (PF) are chemical substances found in plants containing two or more phenol groups. In vitro, $\mathrm{PF}$ have

Abbreviations: FMD, flow-mediated dilatation; LPS, lipopolysaccharide; MCP-1, monocyte chemoattractant protein 1; MIF, macrophage migration inhibitory factor; PF, polyphenols; TLR, Toll-like receptor. 
been shown to inhibit Toll-like receptor (TLR)-mediated inflammatory responses via the MyD88-independent TLR3and TLR4-signalling pathways ${ }^{(13)}$, diminish NF- $\mathrm{KB}$ activation and reduce the production of downstream chemokines and cytokines ${ }^{(13,14)}$. Antioxidative and anti-thrombotic effects have also been reported. Consistent with the anti-atherogenic effects in vitro, PF administration has been shown to attenuate atherosclerotic lesion formation in a variety of animal models $^{(15-18)}$. In human subjects, however, the results of PF administration are heterogeneous ${ }^{(18-22)}$. Whereas a single study did report a beneficial effect of PF on the carotid intima-media thickness in patients at increased cardiovascular risk $^{(23)}$, the true impact of PF ingestion for CVD needs confirmation in larger clinical trials. To date, the bulk of studies observing an effect of PF ingestion is based on epidemiological studies with very few randomised, placebo-controlled trials $^{(24-26)}$.

In the present randomised, placebo-controlled, doubleblind study, we evaluated the impact of daily oral intake of $500 \mathrm{mg}$ of PF-rich extract for 4 weeks on inflammatory markers in thirty-four subjects with a clustering of cardiometabolic risk factors. In addition, we challenged a random subgroup ( $n$ 24) with low-dose endotoxin to also evaluate the effect of the PF-rich extract on the acute inflammatory response.

\section{Methods}

\section{Participants}

In the present study, thirty-four participants were recruited from the outpatient clinic of the Academic Medical Center (Amsterdam, The Netherlands) and through poster advertisement. The subgroup for the endotoxin challenge consisted of twenty-four participants who were randomly selected and asked for consent at the first study visit. Subjects were eligible to participate if two or more of the following criteria were present: a waist circumference of $\geq 102 \mathrm{~cm}$ for men or $\geq 88 \mathrm{~cm}$ for women, TAG levels $\geq 1.69 \mathrm{mmol} / 1$, HDL-cholesterol $\leq 1.03 \mathrm{mmol} / 1$ for men or $\leq 1.29 \mathrm{mmol} / 1$ for women, a systolic blood pressure $\geq 130 \mathrm{mmHg}$, a diastolic blood pressure $\geq 85 \mathrm{mmHg}$ and a glucose level $\geq 6 \cdot 1 \mathrm{mmol} / \mathrm{l}$, according to the Adult Treatment Panel III criteria (attributed to the National Cholesterol Education Program/National Heart, Lung, and Blood Institute) ${ }^{(27)}$. During the study period, participants were instructed to refrain from wine and grape-containing beverages, large amounts of tea, fruit juice and dark chocolate. The use of lipid-lowering medication or antioxidants was not allowed throughout the study. Subjects were excluded if they reported CHD, stroke, malignancies or chronic inflammatory diseases in their medical history. Subjects who were current smokers or known with alcohol abuse were excluded from the study.

\section{Design}

The study was designed as a double-blind, placebo-controlled, randomised, cross-over trial. During the screening visit,
2 weeks before the first study visit, blood was withdrawn for measurement of baseline parameters. Participants were randomised to daily $500 \mathrm{mg}$ PF-rich extract obtained from grapes and apples for 4 weeks or placebo. The PF-rich extract contains a total of thirty-six (poly) phenolic compounds, including flavan-3-ols (monomers and oligomers up to a degree of polymerisation of 10), flavonols (mainly quercetin derivatives and myricetin), chlorogenic acids (5-caffeoylquinic and 4-p-coumaroylquinic acids), stilbenes (trans-resveratrol), dihydrochalcones (phloretin and derivatives) and anthocyanins (delphinidin, cyanidin, petunidin, peonidin and malvidin derivatives). The total amount of (poly) phenolic compounds in the extract is 507 (SD 4) mg/g of power. A detailed analysis section of the PF extract is presented in the supplementary material (available online at http://www.journals.cambridge. org/bjn).

Following a 4-week washout period, participants were switched to placebo or PF-rich extract, respectively. The daily amount of PF-rich extract and placebo was dosed in capsules of $250 \mathrm{mg}$. Participants were asked to take two capsules a day in the morning. At the end of each treatment period, blood was drawn for laboratory testing, including total cholesterol, LDL-cholesterol, HDL-cholesterol, glucose, glycated $\mathrm{Hb}$, C-reactive protein, $\mathrm{Hb}$ and creatinine. Blood pressure was measured and endothelial function (flowmediated dilatation (FMD) by ultrasound) was assessed (see below). All measurements were performed after an overnight fast. At the end of the second treatment period, a subgroup of twenty-four participants (fifteen men and nine women), who consented to additional investigation, was challenged with a low dose of endotoxin derived from Escherichia coli at a dose of $1 \mathrm{ng} / \mathrm{kg}$ of body weight of endotoxin (E. coli lipopolysaccharide (LPS) (O113;H10), 10.00 EU/vial, pharmaceutical development section (PDS) no. 67801, lot no. 67466 (production date 4 January 1997); Cape Cod, Inc./ National Institutes of Health, Bethesda, MD, USA) ${ }^{(28-30)}$ Subsequently, blood was withdrawn from the contralateral antecubital vein at 1, 3, 4, 6 and $8 \mathrm{~h}$. Vital signs were measured at regular intervals, every $15 \mathrm{~min}$ in the first $3 \mathrm{~h}$. Incidence, time and severity of symptoms were recorded by the study physician. FMD measurements were performed at baseline and $4 \mathrm{~h}$ after the LPS challenge. Approval for the present study was obtained from the internal review board of the Academic Medical Center. The study was carried out in accordance with the principles of the Declaration of Helsinki. All participants gave written informed consent.

\section{Laboratory measurements}

Baseline serum concentrations of total cholesterol, HDLcholesterol and TAG were measured in fresh serum samples by standard enzymatic methods (Roche Diagnostics, Basel, Switzerland). LDL-cholesterol concentrations were calculated using the Friedewald formula. Glucose was assessed using the hexokinase method (Gluco-quant, Hitachi 917; Hitachi, Mannheim, Germany). Glycated Hb was measured by HPLC (Reagens; Bio-Rad Laboratories, Veenendaal, The Netherlands) on a Variant II (Bio-Rad Laboratories). Plasma aliquots 
were snap-frozen and stored at $-80^{\circ} \mathrm{C}$ for shipment to the Biomarker Laboratory at the University of Ulm, Germany. Plasma C-reactive protein levels were measured with a high sensitivity latex-enhanced nephelometric assay on a BN II analyser (Dade Behring, Marburg, Germany). TNF- $\alpha$, IL-6, adiponectin, oxidised LDL, MCP-1 and MIF levels were measured using commercially available ELISA kits (R\&D Systems, Abingdon, Oxford, UK). The intra- and inter-assay $\mathrm{CV}$ of quality-control test sera were $<10$ and $<20 \%$, respectively.

\section{Flow-mediated dilatation measurements}

Each patient underwent measurement of FMD of the left brachial artery by B-mode ultrasound imaging using an Acuson Aspen (Siemens, Mountain View, CA, USA) ultrasound system with an $\mathrm{L} 7,5-10 \mathrm{MHz}$ linear array broadband transducer. The FMD of all thirty-four participants was measured after each treatment period. The FMD of the twenty-four participants receiving an additional LPS challenge was also measured $4 \mathrm{~h}$ after the LPS challenge. Patients were instructed to refrain from food, alcohol and any other drink except water before the measurements. A blood pressure cuff was placed on the left forearm from the medial epicondyle downwards. The scan of the left brachial artery started with 1 min of continuous baseline recording, followed by $5 \mathrm{~min}$ of forearm ischaemia, induced by inflating a vascular pressure cuff to $250 \mathrm{mmHg}$. Hyperaemic blood flow was induced by deflating the cuff, followed by $3 \mathrm{~min}$ of continuous ultrasound recording. A semi-automated image analysis of scans was performed off-line by an experienced image analyst using dedicated software (Brachial Analyzer; MIA Vascular Tools, Coralville, IA, USA). Images were blinded for treatment and visit. The average baseline diameter and the maximum post-cuff deflation diameter were used to calculate the percentage of flow-mediated vasodilation (\%FMD), which was defined as follows:

$$
\begin{aligned}
\% \mathrm{FMD}= & 100 \times(\text { maximum post-cuff deflation diameter } \\
& - \text { baseline diameter }) / \text { baseline diameter. }
\end{aligned}
$$

\section{Statistical analysis}

Data are presented as means and standard deviations when normally distributed and as medians with corresponding interquartile ranges for values with a skewed distribution. For the cross-over study, we used a repeated-measures mixed model, with time and treatment as fixed effects. An interaction term of time and treatment was used to test for possible carry-over effects. For the LPS intervention, we calculated the production of circulating mediators after the LPS challenge by the area under the curve from the time of the infusion $(t=0)$ until $6 \mathrm{~h}$ after the start of the challenge $(t=6)$. A paired-samples $t$ test was used for normally distributed values, whereas a Wilcoxon rank test for paired measurements was employed to test for skewed variables. Analyses were performed with
SPSS version 16.0 (SPSS Inc., Chicago, IL, USA). A $P$ value $<0.05$ was defined as statistically significant.

\section{Results}

\section{Baseline characteristics of the participants}

Baseline characteristics of the study participants are listed in Table 1. Of the thirty-four participants, $44 \%$ had two Adult Treatment Panel III factors, whereas $56 \%$ had three Adult Treatment Panel III factors (attributed to the National Cholesterol Education Program/National Heart, Lung, and Blood Institute) ${ }^{(27)}$ of the metabolic syndrome. The prevalence of risk factors was distributed as follows: increased waist circumference $88 \%$, hypertriacylglycerolaemia 50\%, low-HDLcholesterol levels $27 \%$, elevated blood pressure $88 \%$ and elevated plasma glucose levels $29 \%$. The average age was 58.3 (SD 7.9) years. Baseline characteristics of the participants were as follows: BMI 31.9 (SD 4.8$) \mathrm{kg} / \mathrm{m}^{2}$, systolic blood pressure 147 (SD 18.3) $\mathrm{mmHg}$, diastolic blood pressure 89 (SD 9.3) $\mathrm{mmHg}$, total cholesterol 5.6 (SD 1.1) $\mathrm{mmol} / \mathrm{l}$, LDLcholesterol 3.3 (SD $0 \cdot 9) \mathrm{mmol} / \mathrm{l}$, HDL-cholesterol $1 \cdot 3$ (SD 0.4$) \mathrm{mmol} / \mathrm{l}$, TAG $1.76(1.38-3.23) \mathrm{mmol} / \mathrm{l}$, glucose 5.6

Table 1. Markers for cardiovascular risk and inflammation according to the treatment period of all thirty-four participants

\begin{tabular}{|c|c|c|c|c|c|}
\hline & \multicolumn{2}{|c|}{ Placebo } & \multicolumn{2}{|c|}{ Polyphenols } & \multirow[b]{2}{*}{$P$} \\
\hline & Mean & SD & Mean & SD & \\
\hline Age (years) & $58 \cdot 3$ & 7.9 & $58 \cdot 3$ & 7.9 & NS \\
\hline BMI $\left(\mathrm{kg} / \mathrm{m}^{2}\right)$ & 31.9 & 4.9 & 31.6 & $5 \cdot 2$ & NS \\
\hline $\mathrm{SBP}(\mathrm{mmHg})$ & $142 \cdot 5$ & $16 \cdot 8$ & $143 \cdot 0$ & 14.7 & NS \\
\hline $\mathrm{DBP}(\mathrm{mmHg})$ & $86 \cdot 1$ & $10 \cdot 3$ & $87 \cdot 0$ & 8.9 & NS \\
\hline Total cholesterol $(\mathrm{mmol} / \mathrm{l})$ & 5.57 & 1.29 & 5.71 & 1.19 & NS \\
\hline LDL-cholesterol $(\mathrm{mmol} / \mathrm{l})$ & 3.39 & $1 \cdot 16$ & 3.56 & $1 \cdot 11$ & $<0.05$ \\
\hline HDL-cholesterol (mmol/li) & \multirow{2}{*}{\multicolumn{2}{|c|}{1.33}} & \multirow{2}{*}{\multicolumn{2}{|c|}{$1.32 \quad 0.33$}} & NS \\
\hline TAG $(\mathrm{mmol} / \mathrm{l})$ & & & & & NS \\
\hline Median & \multirow{2}{*}{\multicolumn{2}{|c|}{$\begin{array}{c}1 \cdot 76 \\
1 \cdot 33-2 \cdot 39\end{array}$}} & \multicolumn{2}{|c|}{1.88} & \\
\hline Range & & & \multicolumn{2}{|c|}{$1 \cdot 21-2 \cdot 41$} & \\
\hline Glucose $(\mathrm{mmol} / \mathrm{l})$ & \multicolumn{2}{|c|}{$1.33-2.39$} & & & NS \\
\hline Median & \multirow{2}{*}{\multicolumn{2}{|c|}{$\begin{array}{c}6 \cdot 0 \\
5 \cdot 6-6 \cdot 8\end{array}$}} & \multirow{2}{*}{\multicolumn{2}{|c|}{$\begin{array}{c}6.1 \\
5 \cdot 7-6.9\end{array}$}} & \\
\hline Range & & & & & \\
\hline $\mathrm{HbA1C}(\%)$ & \multirow{2}{*}{\multicolumn{2}{|c|}{$5 \cdot 8$}} & \multicolumn{2}{|l|}{$5 \cdot 8$} & NS \\
\hline $\mathrm{CRP}(\mathrm{mg} / \mathrm{l})$ & & & & & NS \\
\hline Median & \multicolumn{2}{|c|}{$2 \cdot 37$} & \multicolumn{2}{|c|}{2.43} & \\
\hline Range & \multirow{2}{*}{\multicolumn{2}{|c|}{$1.36-3.94$}} & \multicolumn{2}{|c|}{$1.19-5.03$} & \\
\hline Oxidised LDL (nmol/ml) & & & & & NS \\
\hline Median & \multicolumn{2}{|c|}{91.0} & \multicolumn{2}{|c|}{91.5} & \\
\hline Range & \multicolumn{2}{|c|}{$77 \cdot 1-118$} & \multicolumn{2}{|c|}{$79-116$} & \\
\hline $\mathrm{IL}-6(\mathrm{pg} / \mathrm{ml})$ & \multirow{2}{*}{\multicolumn{2}{|c|}{1.55}} & & & NS \\
\hline Median & & & \multicolumn{2}{|c|}{1.38} & \\
\hline Range & \multicolumn{2}{|c|}{$1 \cdot 21-1 \cdot 79$} & \multicolumn{2}{|c|}{$1.1-1.83$} & \\
\hline MCP-1 (pg/ml) & \multirow{2}{*}{\multicolumn{2}{|c|}{124}} & & & $<0.05$ \\
\hline Median & & & \multicolumn{2}{|c|}{116} & \\
\hline Range & \multicolumn{2}{|c|}{$105-153$} & \multicolumn{2}{|c|}{$97-135 \cdot 8$} & \\
\hline $\mathrm{MIF}(\mathrm{pg} / \mathrm{ml})$ & & & & & $<0.05$ \\
\hline Median & 281 & & 25 & & \\
\hline Range & $2296-3$ & $852 \cdot 3$ & 1898 & 3972 & \\
\hline Adiponectin $(\mu \mathrm{g} / \mathrm{ml})$ & & & & & NS \\
\hline Median & 8. & & & & \\
\hline Range & $6 \cdot 3-$ & $10 \cdot 8$ & $5 \cdot 3-$ & 11.8 & \\
\hline
\end{tabular}

(Mean values, standard deviations, medians and interquartile ranges)

SBP, systolic blood pressure; DBP, diastolic blood pressure; $\mathrm{HbA1C}$, glycated $\mathrm{Hb}$; CRP, C-reactive protein; MCP-1, monocyte chemoattractant protein 1; MIF, macrophage migration inhibitory factor. 


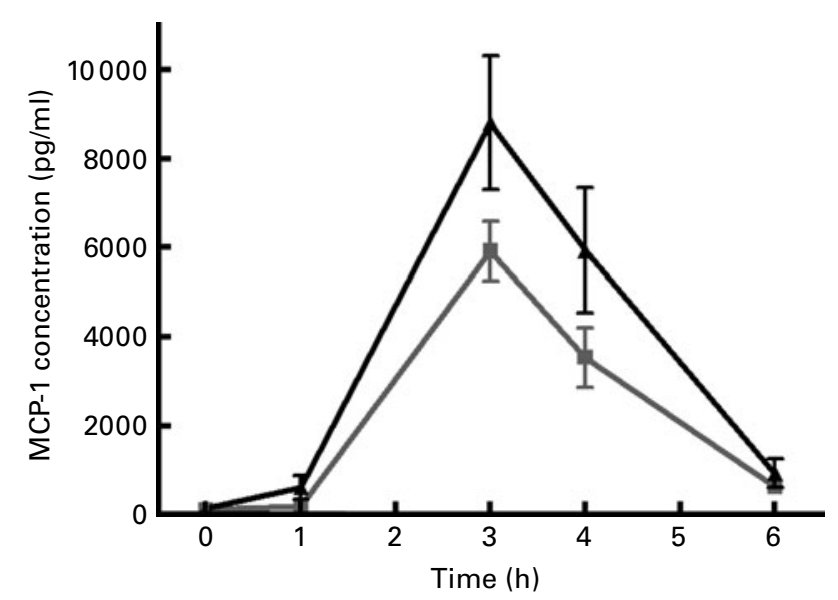

Fig. 1. Area under the curve (over $6 \mathrm{~h}$ of measurement) of monocyte chemoattractant protein 1 (MCP-1). Production of MCP-1 after treatment with polyphenol-rich extract $(\square)$ is significantly lower compared with treatment with placebo

(A); 766.1 v. $1465.5 \mathrm{ng} / \mathrm{ml}(P=0.04)$ (polyphenols $(n 12) v$. placebo $(n 12)$ ). Values are means, with their standard deviations represented by vertical bars.

$(5 \cdot 1-6 \cdot 1) \mathrm{mmol} / \mathrm{l}$, glycated $\mathrm{Hb} 5 \cdot 8$ (SD 0.5$) \%$ and C-reactive protein $2 \cdot 1(1 \cdot 1-2 \cdot 8) \mathrm{mg} / \mathrm{l}$.

\section{Effect of polyphenol-rich extract on inflammatory parameters}

After 4 weeks, BMI and blood pressure were unaffected in both PF-rich extract and placebo groups (Table 1). No significant interaction term was found, indicating that no carry-over effects were present in the present study. We found a statistically significant reduction of 6.5 and $10.8 \%$ for the MCP-1 and MIF levels, respectively, in the PF-rich extract group $v$. placebo. No changes were observed for other markers measured (Table 1). FMD was comparable between the groups (Figs. 1 and 2).

\section{Effects of polyphenol-rich extract on lipopolysaccharide} response

During the final study visit, twenty-four participants received an inflammatory challenge of LPS ( $1 \mathrm{ng} / \mathrm{kg}$ body weight). All participants showed a modest rise in temperature with concomitant side effects including chills, accelerated heart rate and headache. These symptoms did not differ between the PF-rich extract and placebo groups. Total MCP-1 production, expressed as area under the curve up to $6 \mathrm{~h}$, was reduced in the PF-rich extract group compared with placebo. The ensuing peak increases in other measured inflammatory parameters were not significantly different (data not shown). FMD was significantly reduced $4 \mathrm{~h}$ after the LPS challenge (Figs. 1 and 2) in both groups. This attenuation was comparable in both PF-rich extract and placebo groups.

\section{Discussion}

In the present study, we show that the 4-week administration of $500 \mathrm{mg}$ PF-rich extract was associated with a significant, albeit modest, reduction in MCP-1 and MIF levels in subjects with clustered metabolic risk factors. MCP-1 production (area under the curve over $6 \mathrm{~h}$ ) following an LPS challenge was significantly reduced in the PF group compared with placebo, whereas no differences were observed for the other measured inflammatory cytokines. The present findings confirm a modest anti-inflammatory effect of PF-rich extracts in subjects with clustered metabolic risk factors in vivo. Yet, in view of the modest effect on isolated inflammatory parameters, larger studies are needed with prolonged follow-up in order to establish potential clinical relevance of this observation.

\section{Polyphenol and inflammatory markers}

PF-rich extracts from various food sources, including alcoholic beverages made from grapes, have been shown to exert a variety of beneficial effects, including antioxidative, antithrombotic and vasodilatory effects ${ }^{(10,19,31-38)}$. In addition, PF-rich extracts have been reported to reduce the inflammatory response, particularly for TLR3- and TLR4-specific ligands. Since the metabolic syndrome has been associated with a low-grade inflammatory state in which a diet-related, postprandial increase of endotoxins may play a role ${ }^{(39)}$, we focused on patients with clustered metabolic risk factors to assess a potential anti-inflammatory effect of $\mathrm{PF}$ in vivo. Following 4 weeks of PF-rich extract administration, a significant, albeit modest, reduction in circulating levels of MCP-1 and MIF was observed. This finding is in correspondence with previous reports corroborating the anti-inflammatory

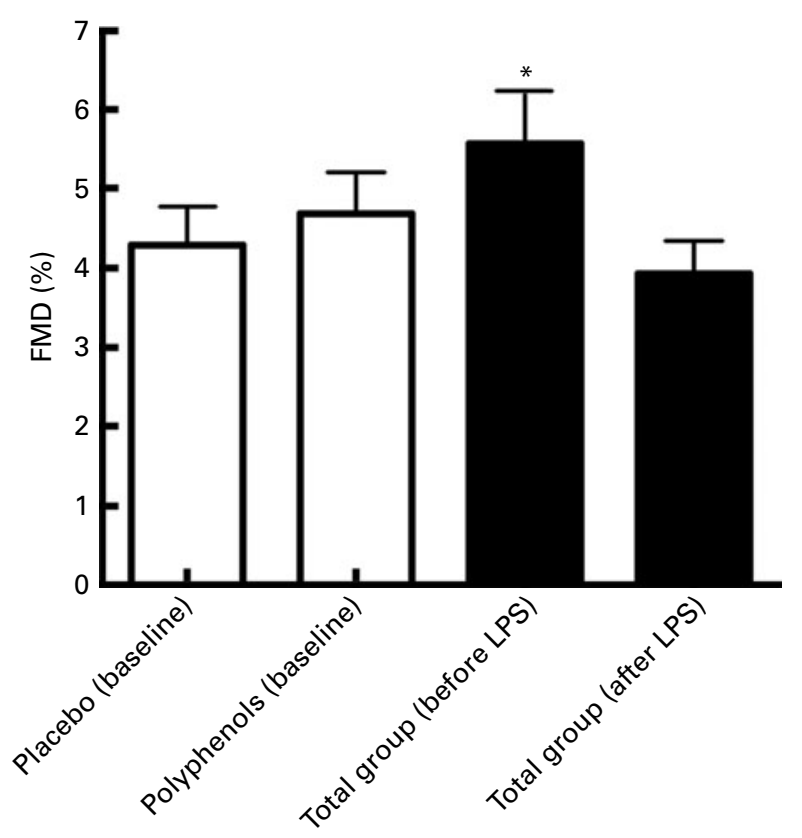

Fig. 2. Flow-mediated dilatation (FMD) of the left brachial artery was not significantly different between the placebo and polyphenol-rich extract groups; 4.28 (SD 0.49) and 4.69 (SD 0.52) ( $P=\mathrm{NS}$ ), respectively ( $n$ 24). FMD did show a significant difference before and $4 \mathrm{~h}$ after the lipopolysaccharide (LPS) infusion in the subgroup; 5.56 (SD 0.7) and 3.84 (SD 0.43) $\left({ }^{*} P<0.05\right), n 14$ Values are means, with their standard deviations represented by vertical bars. 
capacity of PF in animal models ${ }^{(40,41)}$. Similarly, the phenolic compound resveratrol has been shown to inhibit NF- $\mathrm{kB}$ transcription in vitro ${ }^{(13,42)}$. In healthy volunteers, consumption of the PF extract in alcoholic beverages has also been reported to reduce $\mathrm{NF}-\kappa \mathrm{B}$ activation as well as MCP-1 plasma levels during a high-fat diet ${ }^{(43)}$. Both MCP-1 and MIF are considered to be important pro-inflammatory cytokines associated with increased cardiovascular risk. MCP-1 is an important chemokine, attracting monocytes to the sites of endothelial injury, and both MCP-1 serum levels and MCP-1 genotype have been associated with $\mathrm{CVD}^{(44)}$. Mice receiving pharmacological blockade of MCP-1 and mice deficient in MCP-1 develop less atherosclerosis compared with placebo-treated or wild-type mice ${ }^{(45,46)}$. Similarly, MIF has been associated with atherogenesis as well as risk factors associated with the development of metabolic disorders such as obesity and insulin resistance ${ }^{(47)}$. MIF is a pleiotropic cytokine that acts, among others, as a proinflammatory cytokine ${ }^{(48)}$. Both MIF and MCP-1 play a crucial role in the chemotaxis of monocytes into the adipose tissue, which is a key step in the progression towards 'dysfunctional' adipose tissue $^{(47,49,50)}$, characterised by a dysregulation of adipocyte paracrine function. We did not observe a difference in other markers associated with a pro-atherogenic state between the PF-rich extract and placebo groups. Based on the present findings, one might speculate whether PF-rich extracts can have a positive effect on 'dysfunctional' fat tissue in patients with the metabolic syndrome after long-term exposure ${ }^{(10)}$

\section{Polyphenol and the inflammatory reaction following lipopolysaccharide challenge}

Consistent with the effect of the PF-rich extract on the chronic inflammatory state, a challenge with LPS resulted in a reduced MCP-1 area under the curve over $6 \mathrm{~h}$ in the PF-rich extract group compared with placebo. The other measured inflammatory markers did not differ. Monagas et al. ${ }^{(51)}$ recently described that pre-treatment of peripheral blood mononuclear cells with certain phenolic metabolites induced a reduction of TNF- $\alpha$, IL- 6 and IL-1 $\beta$ production after LPS stimulation by more than $80 \%$. Another in vitro study has observed differential effects between the various phenolic compounds that were used. For instance, a reduction of IL-1 $\beta$ could be detected for addition of oleuropein glycoside, present in olive oil, but not for other PF used. No effect was observed on TNF- $\alpha$ or IL- $6^{(52)}$, the latter finding being more similar to the results of the present study. It has been suggested that not all PF have comparable anti-inflammatory characteristics and that different PF have specific anti-inflammatory properties, which could in part explain the heterogeneous results between previously published studies ${ }^{(53)}$.

\section{Limitations}

The results of the present study warrant several comments. First, a treatment period of 4 weeks is a relatively short period and the dose of $500 \mathrm{mg} / \mathrm{d}$ may be suboptimal as bioavailability of many polyphenolic substances has been reported to be poor ${ }^{(54)}$. Thus, large amounts of PF may have to be ingested in order to obtain beneficial effects. It has to be taken in account that this characteristic limits the clinical application of these compounds. Second, the PF-rich extract used is a mixture of compounds, which includes various substances and may even enclose potential non-polyphenolic compounds. This is a first exploratory study to investigate the effect of a PF-rich extract on both acute and chronic inflammatory reactions in obese participants with multiple cardiometabolic risk factors. Markedly, despite the short duration of PF administration as well as the limited number of participants studied, we were able to detect a significant effect on both basal MCP-1 and MIF plasma levels, as well as the acute MCP-1 response following an endotoxin challenge.

\section{Conclusions}

The present study shows that oral ingestion of a PF-rich mixture extracted from apples and grapes decreases circulating pro-inflammatory cytokines, involved in both the pathogenesis of adipocyte dysfunction and atherogenesis. Considering the important role for the infiltration of monocytes into fat tissue and the subsequent inflammatory response, $\mathrm{PF}$ may have a potentially beneficial effect on the development and/or progression of CVD in patients with clustered metabolic risk factors. However, this needs further confirmation in larger trials investigating the optimal dose and the long-term results of $\mathrm{PF}$ administration on the development and progression of CVD.

\section{Acknowledgements}

None of the authors had a conflict of interest. The present study was supported by the Dutch Heart Foundation, The Netherlands (grant no. 2006B88) and Imagination Unlimited BV, Wageningen, The Netherlands. We thank Johan Gort and Raphaël Duivenvoorden for their imaging assistance. The authors' responsibilities were as follows: L. N. B., E. S. G. S., P. G. H., H. V. and J. J. P. K. contributed to the study conception and design; L. N. B., D. F. v. W., H. V. and W. K. were involved in the data acquisition; L. N. B., D. F. v. W., B. A. H. and E. S. G. S. were responsible for the statistical analyses and interpretation of the data; L. N. B., D. F. v. W. and E. S. G. S. helped in writing of the final version of the manuscript; J. J. P. K., P. G. H. and W. K. contributed to the critical revision of the manuscript. Each author contributed significantly to the study and read and approved the final manuscript.

\section{References}

1. Libby P (2002) Atherosclerosis: the new view. Sci Am 286, $46-55$.

2. Koenig W, Khuseyinova N, Baumert J, et al. (2006) Increased concentrations of C-reactive protein and IL- 6 but not IL-18 are independently associated with incident coronary events in middle-aged men and women: results from the MONICA/ KORA Augsburg case-cohort study, 1984-2002. Arterioscler Thromb Vasc Biol 26, 2745-2751.

3. Herder C, Baumert J, Thorand B, et al. (2006) Chemokines and incident coronary heart disease: results from the 
MONICA/KORA Augsburg case-cohort study, 1984-2002. Arterioscler Thromb Vasc Biol 26, 2147-2152.

4. Tuomisto K, Jousilahti P, Sundvall J, et al. (2006) C-reactive protein, interleukin-6 and tumor necrosis factor alpha as predictors of incident coronary and cardiovascular events and total mortality. A population-based, prospective study. Thromb Haemost 95, 511-518.

5. Blake GJ \& Ridker PM (2003) C-reactive protein and other inflammatory risk markers in acute coronary syndromes. J Am Coll Cardiol 41, 4 Suppl. S, 37S-42S.

6. Hlawaty H, Jacob MP, Louedec L, et al. (2009) Leukotriene receptor antagonism and the prevention of extracellular matrix degradation during atherosclerosis and in-stent stenosis. Arterioscler Thromb Vasc Biol 29, 518-524.

7. Ferrante AW Jr (2007) Obesity-induced inflammation: a metabolic dialogue in the language of inflammation. J Intern Med 262, 408-414.

8. Conen D, Rexrode KM, Creager MA, et al. (2009) Metabolic syndrome, inflammation, and risk of symptomatic peripheral artery disease in women. A prospective study. Circulation 120, 1041-1047.

9. Rutter MK, Meigs JB, Sullivan LM, et al. (2004) C-reactive protein, the metabolic syndrome, and prediction of cardiovascular events in the Framingham Offspring Study. Circulation 110, 380-385.

10. Wellen KE \& Hotamisligil GS (2003) Obesity-induced inflammatory changes in adipose tissue. J Clin Invest $\mathbf{1 1 2}$, $1785-1788$.

11. Gustafson B, Hammarstedt A, Andersson CX, et al. (2007) Inflamed adipose tissue: a culprit underlying the metabolic syndrome and atherosclerosis. Arterioscler Thromb Vasc Biol 27, 2276-2283.

12. Kamei N, Tobe K, Suzuki R, et al. (2006) Overexpression of monocyte chemoattractant protein-1 in adipose tissues causes macrophage recruitment and insulin resistance. J Biol Chem 281, 26602-26614.

13. Youn HS, Lee JY, Fitzgerald KA, et al. (2005) Specific inhibition of MyD88-independent signaling pathways of TLR3 and TLR4 by resveratrol: molecular targets are TBK1 and RIP1 in TRIF complex. J Immunol 175, 3339-3346.

14. Kang OH, Jang HJ, Chae HS, et al. (2009) Anti-inflammatory mechanisms of resveratrol in activated HMC-1 cells: pivotal roles of NF-kappaB and MAPK. Pharmacol Res 59, 330-337.

15. Do GM, Kwon EY, Kim HJ, et al. (2008) Long-term effects of resveratrol supplementation on suppression of atherogenic lesion formation and cholesterol synthesis in apo E-deficient mice. Biochem Biophys Res Commun 374, 55-59.

16. Wang Z, Zou J, Cao K, et al. (2005) Dealcoholized red wine containing known amounts of resveratrol suppresses atherosclerosis in hypercholesterolemic rabbits without affecting plasma lipid levels. Int J Mol Med 16, 533-540.

17. Fuhrman B, Volkova N, Coleman R, et al. (2005) Grape powder polyphenols attenuate atherosclerosis development in apolipoprotein E deficient (E0) mice and reduce macrophage atherogenicity. $J$ Nutr 135, 722-728.

18. Perez-Jimenez J \& Saura-Calixto F (2008) Grape products and cardiovascular disease risk factors. Nutr Res Rev 21, 158-173.

19. Dell'Agli M, Busciala A \& Bosisio E (2004) Vascular effects of wine polyphenols. Cardiovasc Res 63, 593-602.

20. Leifert WR \& Abeywardena MY (2008) Cardioprotective actions of grape polyphenols. Nutr Res 28, 729-737.

21. Desch S, Schmidt J, Kobler D, et al. (2010) Effect of cocoa products on blood pressure: systematic review and metaanalysis. Am J Hypertens 23, 97-103.
22. Davidson MH, Maki KC, Dicklin MR, et al. (2009) Effects of consumption of pomegranate juice on carotid intima-media thickness in men and women at moderate risk for coronary heart disease. Am J Cardiol 104, 936-942.

23. Aviram M, Rosenblat M, Gaitini D, et al. (2004) Pomegranate juice consumption for 3 years by patients with carotid artery stenosis reduces common carotid intima-media thickness, blood pressure and LDL oxidation. Clin Nutr 23, 423-433.

24. Basu A, Du M, Sanchez K, et al. (2011) Green tea minimally affects biomarkers of inflammation in obese subjects with metabolic syndrome. Nutrition 27, 206-213.

25. Basu A, Du M, Leyva MJ, et al. (2010) Blueberries decrease cardiovascular risk factors in obese men and women with metabolic syndrome. J Nutr 140, 1582-1587.

26. Jimenez JP, Serrano J, Tabernero M, et al. (2008) Effects of grape antioxidant dietary fiber in cardiovascular disease risk factors. Nutrition 24, 646-653.

27. Grundy SM, Brewer HB Jr, Cleeman JI, et al. (2004) Definition of metabolic syndrome: report of the National Heart, Lung, and Blood Institute/American Heart Association conference on scientific issues related to definition. Circulation 109, 433-438.

28. Birjmohun RS, van Leuven SI, Levels JH, et al. (2007) Highdensity lipoprotein attenuates inflammation and coagulation response on endotoxin challenge in humans. Arterioscler Thromb Vasc Biol 27, 1153-1158.

29. Nieuwdorp M, van Haeften TW, Gouverneur MC, et al. (2006) Loss of endothelial glycocalyx during acute hyperglycemia coincides with endothelial dysfunction and coagulation activation in vivo. Diabetes 55, 480-486.

30. Olszyna DP, De JE, Dekkers PE, et al. (2001) Induction of cell-associated chemokines after endotoxin administration to healthy humans. Infect Immun 69, 2736-2738.

31. Estruch R, Sacanella E, Badia E, et al. (2004) Different effects of red wine and gin consumption on inflammatory biomarkers of atherosclerosis: a prospective randomized crossover trial. Effects of wine on inflammatory markers. Atherosclerosis 175, 117-123.

32. Arts IC \& Hollman PC (2005) Polyphenols and disease risk in epidemiologic studies. Am J Clin Nutr 81, 1 Suppl., 317S-325s

33. Sies H, Schewe T, Heiss C, et al. (2005) Cocoa polyphenols and inflammatory mediators. Am J Clin Nutr 81, 1 Suppl., $304 \mathrm{~S}-312 \mathrm{~S}$

34. Vita JA (2005) Polyphenols and cardiovascular disease: effects on endothelial and platelet function. Am J Clin Nutr 81, 1 Suppl., 292S-297S.

35. Oak MH, El BJ \& Schini-Kerth VB (2005) Antiangiogenic properties of natural polyphenols from red wine and green tea. J Nutr Biochem 16, 1-8.

36. Nestel P (2003) Isoflavones: their effects on cardiovascular risk and functions. Curr Opin Lipidol 14, 3-8.

37. Opie LH \& Lecour S (2007) The red wine hypothesis: from concepts to protective signalling molecules. Eur Heart $J$ 28, 1683-1693.

38. Stoclet JC, Chataigneau T, Ndiaye M, et al. (2004) Vascular protection by dietary polyphenols. Eur J Pharmacol $\mathbf{5 0 0}$ 299-313

39. Erridge C, Attina T, Spickett CM, et al. (2007) A high-fat meal induces low-grade endotoxemia: evidence of a novel mechanism of postprandial inflammation. Am J Clin Nutr 86, 1286-1292.

40. DeFuria J, Bennett G, Strissel KJ, et al. (2009) Dietary blueberry attenuates whole-body insulin resistance in high fatfed mice by reducing adipocyte death and its inflammatory sequelae. J Nutr 139, 1510-1516. 
41. Feng AN, Chen YL, Chen YT, et al. (1999) Red wine inhibits monocyte chemotactic protein-1 expression and modestly reduces neointimal hyperplasia after balloon injury in cholesterol-fed rabbits. Circulation 100, 2254-2259.

42. Manna SK, Mukhopadhyay A \& Aggarwal BB (2000) Resveratrol suppresses TNF-induced activation of nuclear transcription factors NF-kappa B, activator protein-1, and apoptosis: potential role of reactive oxygen intermediates and lipid peroxidation. J Immunol 164, 6509-6519.

43. Blanco-Colio LM, Munoz-Garcia B, Martin-Ventura JL, et al. (2007) Ethanol beverages containing polyphenols decrease nuclear factor kappa-B activation in mononuclear cells and circulating MCP-1 concentrations in healthy volunteers during a fat-enriched diet. Atherosclerosis 192, 335-341.

44. McDermott DH, Yang Q, Kathiresan S, et al. (2005) CCL2 polymorphisms are associated with serum monocyte chemoattractant protein-1 levels and myocardial infarction in the Framingham Heart Study. Circulation 112, 1113-1120.

45. Boring L, Gosling J, Cleary M, et al. (1998) Decreased lesion formation in CCR $2^{-1-}$ mice reveals a role for chemokines in the initiation of atherosclerosis. Nature 394, 894-897.

46. Gu L, Okada Y, Clinton SK, et al. (1998) Absence of monocyte chemoattractant protein-1 reduces atherosclerosis in low density lipoprotein receptor-deficient mice. $\mathrm{Mol}$ Cell $\mathbf{2}$, $275-281$

47. Verschuren L, Kooistra T, Bernhagen J, et al. (2009) MIF deficiency reduces chronic inflammation in white adipose tissue and impairs the development of insulin resistance, glucose intolerance, and associated atherosclerotic disease. Circ Res 105, 99-107.

48. Chen L, Yang G, Zhang X, et al. (2009) Induction of MIF expression by oxidized LDL via activation of NF-kappaB in vascular smooth muscle cells. Atherosclerosis 207, 428-433.

49. Kanda H, Tateya S, Tamori Y, et al. (2006) MCP-1 contributes to macrophage infiltration into adipose tissue, insulin resistance, and hepatic steatosis in obesity. J Clin Invest 116, $1494-1505$.

50. Zhu J, Yong W, Wu X, et al. (2008) Anti-inflammatory effect of resveratrol on TNF-alpha-induced MCP-1 expression in adipocytes. Biochem Biophys Res Commun 369, 471-477.

51. Monagas M, Khan N, Andres-Lacueva C, et al. (2009) Dihydroxylated phenolic acids derived from microbial metabolism reduce lipopolysaccharide-stimulated cytokine secretion by human peripheral blood mononuclear cells. Br J Nutr 102, 201-206.

52. Miles EA, Zoubouli P \& Calder PC (2005) Differential antiinflammatory effects of phenolic compounds from extra virgin olive oil identified in human whole blood cultures. Nutrition 21, 389-394.

53. Miles EA, Zoubouli P \& Calder PC (2005) Effects of polyphenols on human Th1 and Th2 cytokine production. Clin Nutr 24, 780-784.

54. Yang CS, Sang S, Lambert JD, et al. (2008) Bioavailability issues in studying the health effects of plant polyphenolic compounds. Mol Nutr Food Res 52, Suppl. 1, S139-S151. 